\title{
Preclinical efficacy studies of a novel nanoparticle-based formulation of paclitaxel that out-performs Abraxane
}

\author{
Zhongling Feng • Gang Zhao • Lei Yu • David Gough • \\ Stephen B. Howell
}

Received: 19 January 2009 / Accepted: 28 July 2009 / Published online: 15 August 2009

(C) The Author(s) 2009. This article is published with open access at Springerlink.com

\begin{abstract}
Purpose Poly-( $\gamma$-L-glutamylglutamine)-paclitaxel (PGGPTX) is a novel polymer-based formulation of paclitaxel (PTX) in which the PTX is linked to the polymer via ester bonds. PGG-PTX is of interest because it spontaneously forms very small nanoparticles in plasma. In mouse models, PGG-PTX increased tumor exposure to PTX by 7.7-fold relative to that produced by PTX formulated in Cremophor. In this study, the efficacy of PGG-PTX was compared to that of Abraxane, an established nanoparticular formulation of PTX, in three different tumor models.

Methods Efficacy was quantified by delay in tumor growth of NCI H460 human lung cancer, 2008 human ovarian cancer and B16 melanoma xenografts growing in athymic mice following administration of equitoxic doses of PGG-PTX and Abraxane administered on either a single
\end{abstract}

\footnotetext{
Z. Feng $\cdot$ G. Zhao $\cdot$ L. Yu

Biogroup, Nitto Denko Technical Corporation,

501 Via Del Monte, Oceanside, CA 92058, USA

G. Zhao

e-mail: gang_zhao@gg.nitto.co.jp

L. Yu

e-mail: lei_yu@gg.nitto.co.jp

D. Gough

Department of Bioengineering, University of California,

San Diego, La Jolla, CA, USA

S. B. Howell ( $\square)$

Moores UCSD Cancer Center, University of California,

San Diego, 3855 Health Sciences Drive, La Jolla,

CA 92093-0819, USA

e-mail: showell@ucsd.edu
}

dose or every 7 day schedule. Toxicity was assessed by change in total body weight.

Results The efficacy and toxicity of PGG-PTX was shown to increase with dose in the H460 model. PGG-PTX was $\sim 1.5$-fold less potent than Abraxane. PGG-PTX produced statistically significantly greater inhibition of tumor growth than Abraxane in all three tumor models when mice were given single equitoxic doses of drug. When given every 7 days for 3 doses, PGG-PTX produced greater inhibition of tumor growth while generating much less weight loss in mice bearing H460 tumors.

Conclusion PGG-PTX has activity that is superior to that of Abraxane in multiple tumor models. PGG-PTX has the potential to out-perform Abraxane in enhancing the delivery of PTX tumors while at the same time further reducing the toxicity of both single dose and weekly treatment regimens.

Keywords Paclitaxel - Abraxane - Drug delivery · Lung cancer $\cdot$ Ovarian cancer $\cdot$ Melanoma
Abbreviations
MTD Maximum tolerated dose
PGA Poly(L-glutamic acid)
PGG Poly(L- $\gamma$-glutamylglutamine)
PGG-PTX $70 \mathrm{kDa}$ PGA to which both additional gluta- mine side chains and PTX have been added
PTX Paclitaxel

\section{Introduction}

Paclitaxel (PTX) is effective for the treatment of a wide variety of cancers but, because of its limited solubility in water, it is currently formulated as a concentrated solution 
containing $6 \mathrm{mg}$ PTX/ml Cremophor EL and ethanol (50\% $\mathrm{v} / \mathrm{v})$ that must be further diluted before administration. Cremaphor EL is a biologically and pharmacologically active compound and its use is associated with acute hypersensitivity reactions $[4,15]$. Many investigators have tried to develop PTX formulations using liposomes, microspheres, micelles, nanoparticle, prodrugs, and polymer-drug conjugates [4, 24]. One of these, paclitaxel protein-bound particles for injection (Abraxane), is based on the use of a nanoparticle made from a mixture of paclitaxel and albumin and is now marketed for the treatment of breast cancer. However, the fractional improvement in breast cancer progression-free survival was quite modest [5]. Another, CT-2103 (Xyotax), takes advantage of the fact that paclitaxel can be made more soluble by conjugating it to the water-soluble polymer poly(L-glutamic acid) $[9,10$, 22]. Like Abraxane, CT-2103 exhibits reduced toxicity and increased efficacy in preclinical models including paclitaxel-resistant tumors $[1-3,6,7,11-13,16,18,25,26]$. However, despite favorable phase II clinical trial results $[13,21,23]$, three randomized phase III trials in patients with non-small cell lung cancer failed to demonstrate an improvement in either progression-free or overall survival $[8,17,19]$ and CT-2103 has not yet received marketing approval.

We have developed a novel formulation of PTX that utilizes both the ability of a polymer to enhance solubility and to form nanoparticles with the goal of even further enhancing the therapeutic efficacy of PTX. The polymer backbone of this formulation consists of poly(L-glutamic acid) to which a glutamate side chain has been added to each monomer in the polymer to create poly(L-glutamylglutamate) (PGG). As shown in Fig. 1, these both increases the number of $-\mathrm{COOH}$ groups available to interact with water molecules so that solubility is enhanced and approximately doubles the molecular weight of the polymer. When PTX is conjugated to this polymer to an extent of $35 \%$ (w/w) to create poly-( $\gamma$-L-glutamylglutamine)-paclitaxel (PGGPTX), the tendency of the hydrophobic PTX molecules to interact with each other causes the polymer to collapse to form a nanoparticle of $\sim 20 \mathrm{~nm}$ in aqueous solutions as determined by dynamic light scattering. This particle is considerably smaller than the nanoparticles that make up Abraxane (80-120 nm) which may favor both the exit of this particle from the vascular compartment once it reaches a tumor, and movement through the tumor extracellular fluid.

The efficacy of PGG-PTX has now been tested in a panel of murine and human tumor xenografts in which the activity of PGG-PTX was directly compared to that of Abraxane when the two drugs were given at equitoxic doses. We report here that PGG-PTX out-performs Abraxane in several of these models on a single dose schedule,

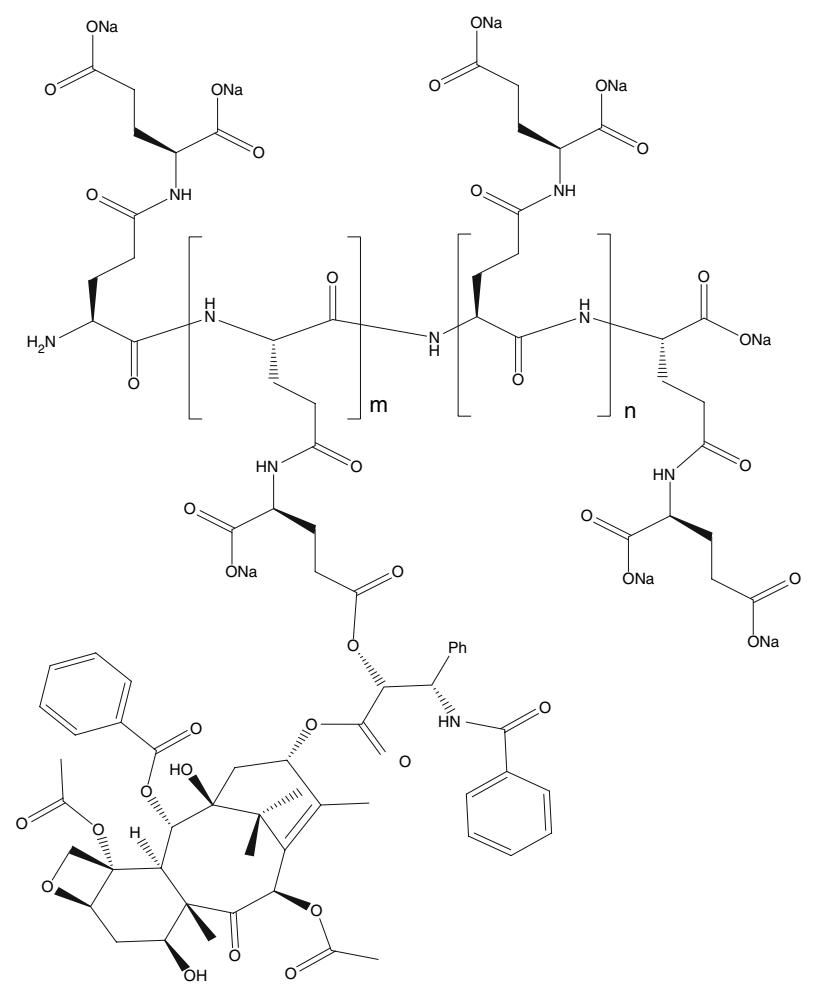

Fig. 1 Structure of PGGA-PTX, a random ester conjugate of poly(L- $\gamma$-glutamylglutamine) and paclitaxel. There are approximately 5 non-conjugated monomer glutamylglutamine units per paclitaxelconjugated monomer glutamylglutamine unit

and that PGG-PTX is substantially less toxic than Abraxane when used on a multidose schedule.

\section{Materials and methods}

Chemicals and reagents

Poly-( $\gamma$-L-glutamylglutamine)-paclitaxel (PGG-PTX) was synthesized from commercially available poly(L-glutamic acid) sodium salt with a molecular weight of $24,880 \mathrm{Da}$. L-glutamic acid di-t.-butyl ester hydrochloride was linked to each monomer in the polymer using a coupling reagent. PTX was then conjugated to the poly(L-glutamylglutamate) using $N$-(3-dimethylaminopropyl)- $N$-ethylcarbodiimide. The PTX content of PGG-PTX was quantified and found to be $35.8 \%(\mathrm{w} / \mathrm{w})$. The PGG-PTX was dissolved in saline at concentration of $17.5 \mathrm{mg} \mathrm{PTX} / \mathrm{ml}(50 \mathrm{mg}$ total weight $/ \mathrm{ml})$. Fresh stock solutions were prepared on the day of injection. Commercially available Abraxane (Los Angeles, CA) was dissolved in saline at concentration of $80 \mathrm{mg} / \mathrm{ml}$. All other chemicals and reagents were obtained from Sigma Chemical Co. (St. Louis, MO), Invitrogen/Life Technologies, Inc. (Carlsbad, CA), Millipore Coorporation (Temecula, CA), or Hyclone (Logan, UT). 


\section{Cell culture}

NCI-H460 and 2008 cells were maintained in RPMI-1640 supplemented with $10 \%$ fetal bovine serum containing $100 \mathrm{U} / \mathrm{ml}$ penicillin and $100 \mu \mathrm{g} / \mathrm{ml}$ streptomycin. B16-F0 cells were grown in DMEM supplemented with $10 \%$ bovine serum, $100 \mathrm{U} / \mathrm{ml}$ penicillin and $100 \mu \mathrm{g} / \mathrm{ml}$ streptomycin. Cells were split $48 \mathrm{~h}$ before inoculation into mice so that they were in $\log$ phase growth when harvested.

\section{Efficacy studies}

NCI-H460 and 2008 cells were suspended in serum-free RPMI 1640 medium, and B16-F0 cells suspended in serumfree DMEM medium, and then injected subcutaneously. The inoculum size per site was $4 \times 10^{6}$ cells for the NCI-H460 cells, $2 \times 10^{6}$ for the 2008 cells, and $0.5 \times 10^{6}$ for the B16 cells. NCI-H460 and B16 cells were injected over each shoulder and each hip of 6- to 8-week-old female athymic nude (nu/nu) mice (Charles River lab, Willington, MA) (4 sites per mouse). The 2008 cells were injected only over both shoulders. Tumors were allowed to grow until they reached an average volume of $30-40 \mathrm{~mm}^{3}$. Tumor size in $\mathrm{mm}^{3}$ was estimated from the formula $\left(w^{2} \times l\right) / 2$ where " $l$ " is the longest diameter of the tumor and " $w$ " is the diameter perpendicular to the longest diameter measured in millimeters. Tumor-bearing animals were then randomly sorted into 3 groups of 6-10 mice each. Both Abraxane and PGG-PTX were administered as a single IP injection. Control animals were injected with saline in a volume equivalent to the volume of PGG-PTX. The MTD was defined as the dose that produced $10 \%$ weight loss. Mice were weighed daily and tumor volumes were measured every other day until total tumor burden reached $1.5 \mathrm{~cm}^{3}$ at which time mice were killed. All in vivo studies were performed at the animal facility of University of California San Diego in accordance with institutional guidelines set out by Institutional Animal Care and Use Committee (IACUC).

\section{Statistics}

The slope of the regression of log (tumor volume) on time was determined for each individual tumor and the mean of the slopes of all tumors within a group was compared using the Student's $t$ test.

\section{Results}

Activity of PGG-PTX in NCI-H460 human lung cancer xenograft model as a function of dose

The dose-effect relationship for PGG-PTX was explored in mice bearing NCI-H460 lung cancer xenografts. Mice were

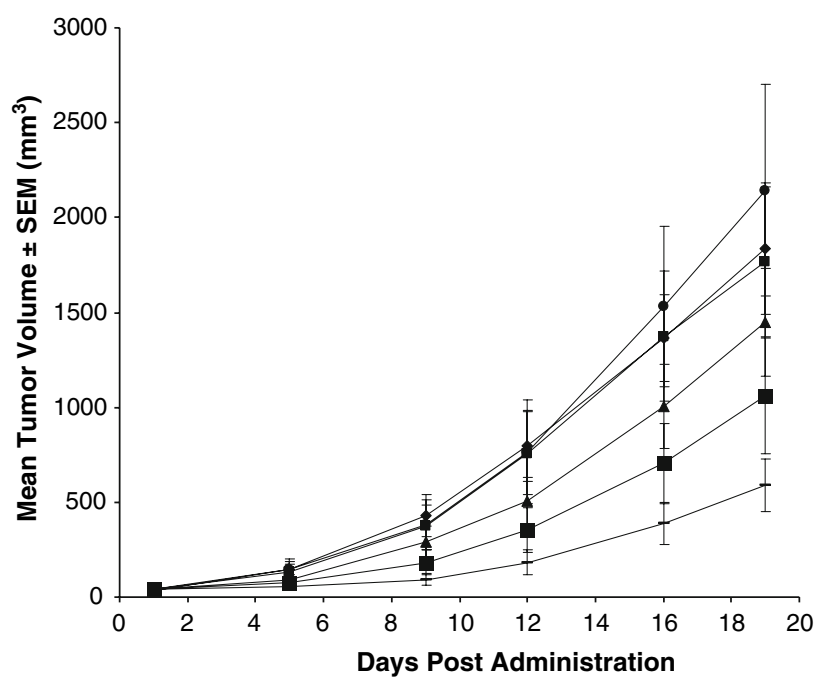

Fig. 2 Tumor growth delay as a function of PGG-PTX dose in mice bearing NCI-H460 tumors. Saline controls (filled circle), PGG-PTX 100 (filled diamond), PGG-PTX 150 (filled square), PGG-PTX 200 (filled triangle), PGG-PTX 250 (filled square), PGG-PTX $300 \mathrm{mg}$ $\mathrm{PTX} / \mathrm{kg}$ (thick line). Vertical bars \pm SEM

inoculated with NCI-H460 cells SC at 4 sites and randomly assigned to control or experimental groups. A single dose of PGG-PTX was administered IP when average tumor volume was $30-40 \mathrm{~mm}^{3}$. Figure 2 shows that there was a welldefined progressive increase in tumor growth inhibition as the dose of PGG-PTX was increased from 100 to $300 \mathrm{mg}$ $\mathrm{PTX} / \mathrm{kg}$. To assess the statistical significance of this difference, a plot was made for each individual tumor of the log of the tumor volume as a function of time. The slope of this curve was then determined and the mean and standard error of the slopes of each group was calculated. The mean values for each group were compared to each other using the Student's $t$ test. This analysis demonstrated that a significant delay in tumor growth was first detected at a dose of $150 \mathrm{mg}$ $\mathrm{PTX} / \mathrm{kg}(P=0.042)$, and that the significance of the difference relative to the untreated mice increased progressively with dose with $P$ values being 0.006 at $200 \mathrm{mg}$ PTX $/ \mathrm{kg}$, 0.0004 at $250 \mathrm{mg}$ PTX $/ \mathrm{kg}$ and $3.6 \times 10^{-7}$ at $300 \mathrm{mg}$ PTX $/ \mathrm{kg}$. The acute weight loss over the first week after dosing also increased progressively with dose ranging from $1 \%$ at a dose of $150 \mathrm{mg}$ PTX/kg to $8 \%$ at a dose of $250 \mathrm{mg}$ PTX/kg. Thus, PGG-PTX exhibited significant antitumor activity in this model even at a dose that caused minimal weight loss, and both its antitumor activity and toxicity increased in a well-defined dose-dependent manner.

Relative efficacy of PGG-PTX and Abraxane in the NCI-H460 human non-small cell lung cancer xenograft model

The relative efficacy of PGG-PTX and Abraxane was assessed by comparing the effect of a single maximum 

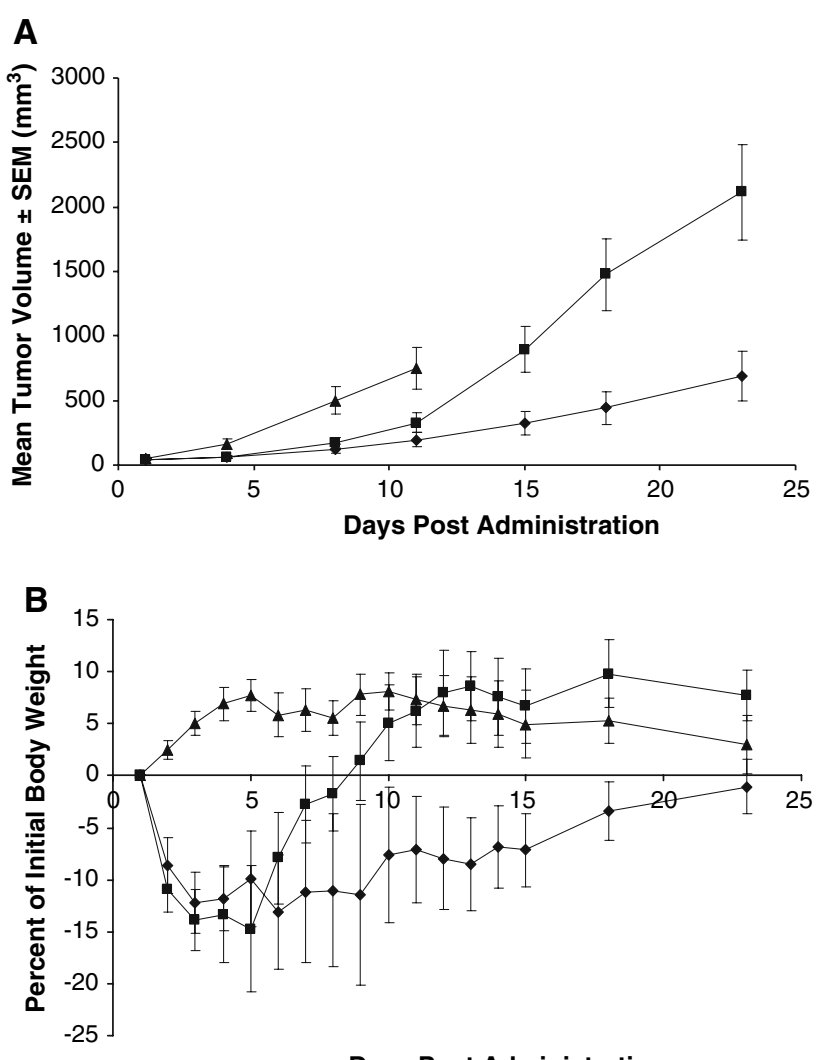

Days Post Administration

Fig. 3 Tumor volume and weight loss as a function of time in mice bearing NCI-H460 tumors treated with a single IP dose of either saline (filled triangle), PGG-PTX (300 mg PTX/kg) (filled diamond) or Abraxane (250 mg PTX/kg) (filled square). Vertical bars $\pm \mathrm{SEM}$

tolerated dose of either drug administered by the IP route on tumor growth delay and weight loss; mice treated with saline alone served as controls. In nu/nu mice bearing NCIH460 tumors, the MTD was $300 \mathrm{mg}$ PTX/kg for PGG-PTX and $250 \mathrm{mg}$ PTX/kg for Abraxane. As shown in Fig. 3a, PGG-PTX produced a greater degree of inhibition of tumor growth than Abraxane. The statistical analysis demonstrated that PGG-PTX produced significant inhibition of tumor growth relative to that in the control mice $(P=0.001)$ but Abraxane did not $(P=0.92)$. At doses that produced equivalent degrees of acute weight loss, the tumor growth delay produced by PGG-PTX was superior to that attained with Abraxane $(P=0.00002)$. The final estimated tumor volume on day 23 in the mice treated with PGGPTX was only $41 \%$ of that in the mice treated with Abraxane.

Figure $3 \mathrm{~b}$ shows that Abraxane at a dose of $250 \mathrm{mg} / \mathrm{kg}$ produced a $15 \pm 6 \%$ (SEM) loss of weight with a nadir on day 5. PGG-PTX at a dose of $300 \mathrm{mg}$ PTX/kg produced the same degree of weight loss $(13 \pm 6 \%)$ with a nadir day 6 . No animals died of toxicity in the PGG-PTX group whereas there was 1 toxic death among the 6 animals in the Abraxane group. However, while both drugs produced equivalent acute reductions in body weight, weight recovery was more rapid following administration of Abraxane. Mice treated with Abraxane regained their initial body weight by day $8-9$, whereas mice treated with PPG-PTX required $>20$ days. This difference is consistent with the observation that PGG-PTX has a much longer plasma halflife (293 h) in mice than Abraxane (19 h) in the rat [26, 27], and suggests that the PTX delivered by PGG-PTX remains in both the tumor and normal tissues for very prolonged periods of time relative to that delivered by Abraxane.

Relative efficacy of PGG-PTX and Abraxane in the human ovarian 2008 xenograft model

The 2008 ovarian cancer model has been widely used for the testing of novel polymeric drug delivery systems [14, 20]. The sensitivity of nu/nu mice bearing these tumors was slightly different than in mice bearing NCI-H460 tumors, and in this model the MTD for Abraxane was only $200 \mathrm{mg}$ PTX/kg. As shown in Fig. 4a, both the PGG-PTX at $300 \mathrm{mg}$ PTX/kg and Abraxane at a dose of $200 \mathrm{mg}$ PTX/kg produced statistically significant degrees of tumor growth inhibition relative to the growth of tumors in control mice $(P=0.006$ and 0.05 , respectively). However, the magnitude of the inhibition was substantially greater for PGGPTX than for Abraxane and this difference between the effect of the two drugs was significant $(P=0.025)$. The final estimated tumor volume on day 91 in the mice treated with PGG-PTX was only $25 \%$ of that in the mice treated with Abraxane. As shown in Fig. 4b, these doses were equitoxic to each other as assessed by the nadir of weight loss which was $10 \pm 1 \%$ (SEM) for PGG-PTX and $7 \pm 3 \%$ (SEM) for Abraxane. As was observed in the NCI-H460 model, body weight recovered more rapidly in mice treated with Abraxane than in those who received PGG-PTX consistent with the difference in the plasma half-lives of these two drugs. There was also a difference in body weights after day 60 that could not be attributed to different numbers of mice surviving in the 3 groups and whose etiology is not currently apparent.

Relative efficacy of PGG-PTX and Abraxane in the murine B16 melanoma model

The murine B16 tumor is another model extensively used for the preclinical assessment of novel drug delivery systems. In order to permit direct comparisons in the same species of mouse, the B16 tumors were also grown in nu/nu mice. In mice bearing B16 tumors, the MTD of PGG-PTX (350 mg PTX/kg) was higher than in the same mice bearing NCI-H460 and 2008 tumors but they were more sensitive to Abraxane whose MTD in this model was just $150 \mathrm{mg}$ PTX/kg. As shown in Fig. 5a, Abraxane at this 

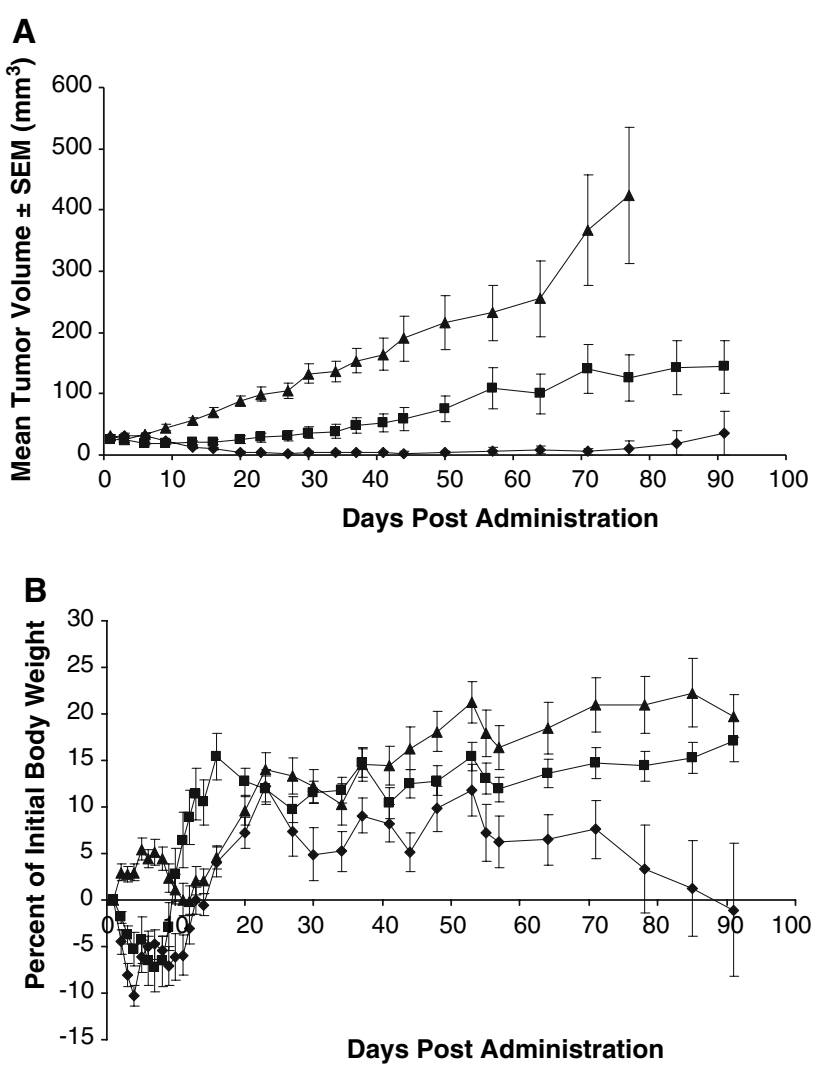

Fig. 4 Tumor volume and weight loss as a function of time in mice bearing human ovarian carcinoma 2008 xenografts treated with a single IP dose of either saline (filled triangle), PGG-PTX (300 mg PTX/ $\mathrm{kg}$ ) (filled diamond) or Abraxane (200 mg PTX/kg) (filled square). Vertical bars \pm SEM

dose produced no detectable inhibition of tumor growth. In contrast, a dose of PGG-PTX that produced approximately the same degree of acute weight loss demonstrated significant tumor growth delay relative to untreated controls $(P=0.0002)$ and relative to mice treated with Abraxane $(P=0.02)$. The final estimated tumor volume on day 9 in the mice treated with PGG-PTX was only $43 \%$ of that in the mice treated with Abraxane. As shown in Fig. 5b, Abraxane at a dose of $150 \mathrm{mg} / \mathrm{kg}$ produced $7 \pm 2 \%$ (SEM) loss of weight with a nadir on day 3. PGG-PTX at a dose of $350 \mathrm{mg}$ PTX/kg produced the same degree of weight loss $[7 \pm 3 \%(\mathrm{SEM})]$ and the nadir also occurred on day 3 . However, the rate of recovery of body weight was slower after this dose of PGG-PTX than it was following Abraxane at $150 \mathrm{mg} / \mathrm{kg}$.

Relative efficacy of PGG-PTX and Abraxane in the NCI-H460 model using a multidose schedule

PTX and Abraxane can be used on either a single dose schedule or a weekly schedule during the treatment of cancer in patients. To compare the relative efficacy of PGG-
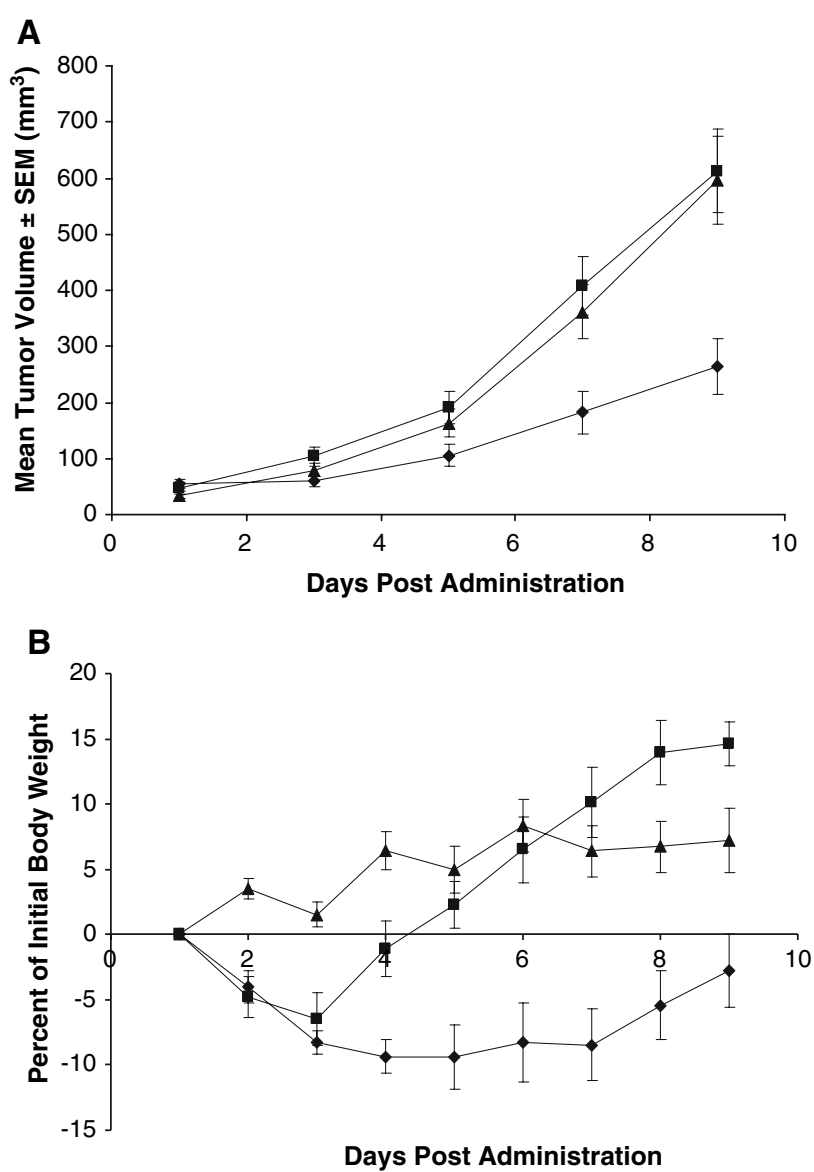

Fig. 5 Tumor volume and weight loss as a function of time in mice bearing murine B16 melanomas treated with a single IP dose of either saline (filled triangle), PGG-PTX (350 mg PTX/kg) (filled diamond) or Abraxane (150 mg PTX/kg) (filled square). Vertical bars \pm SEM

PTX and Abraxane on a weekly schedule, mice inoculated with NCI-H460 tumors were treated with equitoxic doses of each drug every 7 days for 3 doses. Preliminary studies demonstrated that a single IV dose of PGG-PTX at a dose of $140 \mathrm{mg} \mathrm{PTX} / \mathrm{kg}$ was equitoxic with Abraxane at a dose of $40 \mathrm{mg}$ PTX $/ \mathrm{kg}$ with respect to weight loss, and these doses were selected for the multidose schedule. Figure $6 \mathrm{a}$ shows that PGG-PTX was more effective than Abraxane at slowing tumor growth on this multidose schedule $(P=0.02)$. However, the major difference between the two drugs was the toxicity. On this multidose schedule, Abraxane turned out to be much more toxic than PGG-PTX (Fig. 6b). The $140 \mathrm{mg}$ PTX/kg dose of PGG-PTX produced only $7 \pm 3 \%$ (SEM) weight loss by day 29 whereas Abraxane produced a progressive weight loss with each additional dose that resulted in a weight loss of $19 \pm 2 \%$ (SEM) of initial body weight. Thus, on this schedule, the therapeutic ratio of PGG-PTX was substantially better than that of Abraxane. 

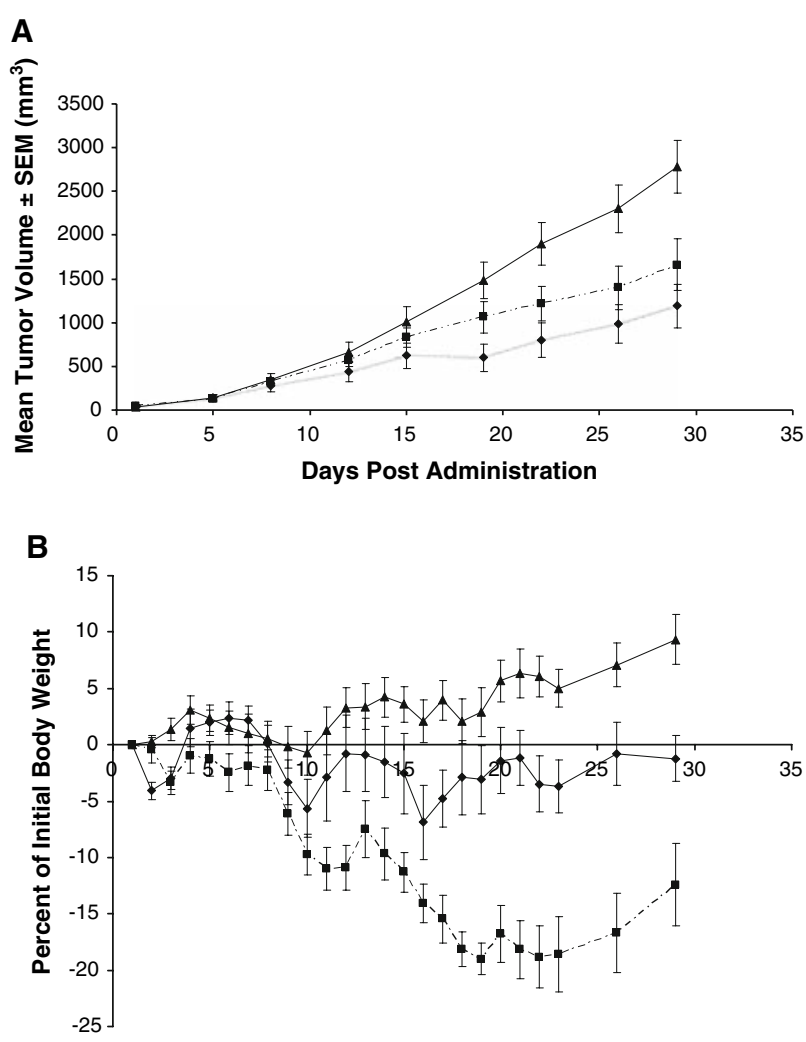

Days Post Administration

Fig. 6 Tumor volume and weight loss as a function of time in mice bearing NCI-H460 lung cancers treated every 7 days for 3 doses with either saline (filled triangle), PGG-PTX (140 mg PTX/kg) (filled diamond) or Abraxane (40 mg PTX/kg) (filled square). Vertical bars \pm SEM

\section{Discussion}

Poly-( $\gamma$-L-glutamylglutamine)-paclitaxel (PGG-PTX) is of interest as a novel drug delivery system for PTX for several reasons. First, it is unique in being a polymer-based system that spontaneously forms nanoparticles in aqueous environments. Ongoing molecular modeling studies suggest that the hydrophobic interactions between the PTX molecules randomly distributed along the PGG polymer cause collapse of the polymer strand to form a particle with a hydrophilic surface and a hydrophobic core. Second, the size of the nanoparticles as determined by dynamic light scattering is substantially smaller than those in the Abraxane formulation ( $\sim 20$ vs. $80-120 \mathrm{~nm}$ ). The large difference in size is likely to translate into important differences in the behavior of the drugs both in the plasma and in tissues. Third, pharmacokinetic studies in nu/nu mice bearing NCI-H460 tumors indicate that conjugation of PTX to the PGG polymer increased plasma and tumor $C_{\max }$, prolonged plasma half-life and the period of accumulation in tumor, and reduced washout from the tumor. The plasma exposure to total taxane produced by PGG-PTX, measured over the first $340 \mathrm{~h}$ after injection, was 23-fold greater than that produced by unconjugated PTX, and the tumor exposure was increased by a factor of 7.7-fold [27]. In contrast, Abraxane increased tumor exposure by only $33 \%$ relative to that attained with Cremophor-based paclitaxel (3632 vs. $2739 \mathrm{nCi} \mathrm{h} / \mathrm{g}$ ) in the MX-1 xenograft model reported by Desai et al. [3]. The results of the current study provide an additional reason for interest in PGG-PTX. In all 3 of the models tested in this study in which a single dose of drug was used, PGG-PTX out-performed Abraxane with respect to inhibition of tumor growth when both drugs were given at doses that produced similar degrees of acute weight loss. In addition, the magnitude of the increase in effectiveness was quite substantial and statistically significant in all 3 models. Perhaps even more importantly, when given every 7 days for 3 doses, PGG-PTX produced greater inhibition of tumor growth while at the same time causing much less toxicity than Abraxane. Even while being less effective, the $40 \mathrm{mg}$ PTX $/ \mathrm{kg}$ dose of Abraxane produced progressive weight loss and was not tolerated on a weekly schedule.

Part of the explanation for the difference in efficacy of PGG-PTX and Abraxane, and for the delayed recovery of body weight in the mice treated with a single dose of PGG-PTX, likely lies in their quite different pharmacokinetics both in the plasma and in normal and malignant tissues. PGG-PTX was found to be somewhat less potent than Abraxane with respect to acute toxicity when given on a single dose schedule. Whereas the MTD for PGGPTX was 300-350 mg PTX/kg in all 3 models, the single dose MTD for Abraxane varied from $150 \mathrm{mg}$ PTX/kg in the B16 model to $250 \mathrm{mg}$ PTX/kg in the NCI H460 lung cancer model. It appears that the type of tumor modulates the toxicity of Abraxane to a greater extent than that of PGG-PTX, an effect most likely related to the specific vascular anatomy of each tumor type. The plasma half-life of Abraxane in mice has not been reported but in the rat it was found to be $19.0 \mathrm{~h}$; the plasma clearance was $517 \mathrm{ml} /$ $\mathrm{h} / \mathrm{kg}$ [26]. In contrast, in pharmacology studies carried out in nu/nu mice bearing NCI H460 tumors executed in parallel with the efficacy studies reported here, the plasma half-life of PGG-PTX was found to be $296.2 \mathrm{~h}$ and the clearance only $11.5 \mathrm{ml} / \mathrm{h} / \mathrm{kg}$ [27]. Whereas Abraxane increased the total tumor exposure to PTX by only $33 \%$ above that produced by an equitoxic dose of PTX formulated as Taxol in the MX1 tumor model [3], PGG-PTX increased exposure for the NCI H460 tumors by a factor of 7.7-fold in the NCI H460 model [27]. Studies directly comparing the tumor exposure to PTX following injection of equitoxic doses of PGG-PTX and Abraxane in the same tumor model have yet to be performed. Nevertheless, these data are consistent with the concept that PGGPTX is more efficient at targeting PTX to tumors than 
Abraxane largely due to differences in their pharmacokinetics.

Abraxane has an established role in the treatment of breast cancer. We conclude from these studies that PGGPTX has the potential to out-perform Abraxane in enhancing the delivery of PTX to such tumors while at the same time further reducing the toxicity of both single dose and weekly treatment regimens.

Acknowledgments We thank Brian Reuter and Ho Lim Fung for technical assistance. Financial support for this work was provided by UC Discovery grant bio06-10568 and the Nitto Denko Technical Corporation. This work was supported by a public-private grant program operated by the University of California in which the University and the Nitto Denko Technical Corp each provide half of the funding. Drs. Xinghe Wang, Gang Zhao, Sang Van, Nan Jiang and Lei Yu are employees of Nitto Denko. Drs. David Vera and Stephen B. Howell are employees of the University of California, San Diego who receive research support under this grant. Drs. Vera and Howell have also served as consultants to Nitto Denko in the past.

Open Access This article is distributed under the terms of the Creative Commons Attribution Noncommercial License which permits any noncommercial use, distribution, and reproduction in any medium, provided the original author(s) and source are credited.

\section{References}

1. Auzenne E, Donato NJ, Li C, Leroux E, Price RE, Farquhar D, Klostergaard J (2002) Superior therapeutic profile of poly-L-glutamic acid-paclitaxel copolymer compared with taxol in xenogeneic compartmental models of human ovarian carcinoma. Clin Cancer Res 8:573

2. Calvo P, Gouritin B, Chacun H, Desmaele D, D'Angelo J, Noel JP, Georgin D, Fattal E, Andreux JP, Couvreur P (2001) Long-circulating PEGylated polycyanoacrylate nanoparticles as new drug carrier for brain delivery. Pharm Res 18:1157

3. Desai N, Trieu V, Yao Z, Louie L, Ci S, Yang A, Tao C, De T, Beals B, Dykes D, Noker P, Yao R, Labao E, Hawkins M, Soon-Shiong P (2006) Increased antitumor activity, intratumor paclitaxel concentrations, and endothelial cell transport of cremophor-free, albumin-bound paclitaxel, ABI-007, compared with cremophor-based paclitaxel. Clin Cancer Res 12:1317

4. Gelderblom H, Verweij J, Nooter K, Sparreboom A (2001) Cremophor EL: the drawbacks and advantages of vehicle selection for drug formulation. Eur J Cancer 37:1590

5. Gradishar WJ, Tjulandin S, Davidson N, Shaw H, Desai N, Bhar P, Hawkins M, O'Shaughnessy J (2005) Phase III trial of nanoparticle albumin-bound paclitaxel compared with polyethylated castor oil-based paclitaxel in women with breast cancer. J Clin Oncol 23:7794

6. Kim SC, Yu J, Lee JW, Park ES, Chi SC (2005) Sensitive HPLC method for quantitation of paclitaxel (Genexol in biological samples with application to preclinical pharmacokinetics and biodistribution. J Pharm Biomed Anal 39:170

7. Langer CJ (2004) CT-2103: emerging utility and therapy for solid tumours. Expert Opin Investig Drugs 13:1501

8. Langer CJ, O’Byrne KJ, Socinski MA, Mikhailov SM, Lesniewski-Kmak K, Smakal M, Ciuleanu TE, Orlov SV, Dediu M, Heigener D, Eisenfeld AJ, Sandalic L, Oldham FB, Singer JW, Ross HJ (2008) Phase III trial comparing paclitaxel poliglumex (CT-2103, PPX) in combination with carboplatin versus standard paclitaxel and carboplatin in the treatment of PS 2 patients with chemotherapy-naive advanced non-small cell lung cancer. J Thorac Oncol 3:623

9. Li C, Ke S, Wu QP, Tansey W, Hunter N, Buchmiller LM, Milas L, Charnsangavej C, Wallace S (2000) Tumor irradiation enhances the tumor-specific distribution of poly(L-glutamic acid)-conjugated paclitaxel and its antitumor efficacy. Clin Cancer Res 6:2829

10. Li C, Newman RA, Wu QP, Ke S, Chen W, Hutto T, Kan Z, Brannan MD, Charnsangavej C, Wallace S (2000) Biodistribution of paclitaxel and poly(L-glutamic acid)-paclitaxel conjugate in mice with ovarian OCa-1 tumor. Cancer Chemother Pharmacol 46:416

11. Li C, Price JE, Milas L, Hunter NR, Ke S, Yu DF, Charnsangavej C, Wallace S (1999) Antitumor activity of poly(L-glutamic acid)paclitaxel on syngeneic and xenografted tumors. Clin Cancer Res 5:891

12. Li C, Yu D, Inoue T, Yang DJ, Milas L, Hunter NR, Kim EE, Wallace S (1996) Synthesis and evaluation of water-soluble polyethylene glycol-paclitaxel conjugate as a paclitaxel prodrug. Anticancer Drugs 7:642

13. Lin NU, Parker LM, Come SE, Burstein HJ, Haldoupis M, Ryabin N, Gelman R, Winer EP, Shulman LN (2007) Phase II study of CT-2103 as first- or second-line chemotherapy in patients with metastatic breast cancer: unexpected incidence of hypersensitivity reactions. Invest New Drugs 25:369

14. Lin X, Zhang Q, Rice JR, Stewart DR, Nowotnik DP, Howell SB (2004) Improved targeting of platinum chemotherapeutics. the antitumour activity of the HPMA copolymer platinum agent AP5280 in murine tumour models. Eur J Cancer 40:291

15. Longnecker SM, Donehower RC, Cates AE, Chen TL, Brundrett RB, Grochow LB, Ettinger DS, Colvin M (1987) High-performance liquid chromatographic assay for taxol in human plasma and urine and pharmacokinetics in a phase I trial. Cancer Treat Rep $71: 53$

16. Multani AS, Li C, Ozen M, Yadav M, Yu DF, Wallace S, Pathak S (1997) Paclitaxel and water-soluble poly (L-glutamic acid)-paclitaxel, induce direct chromosomal abnormalities and cell death in a murine metastatic melanoma cell line. Anticancer Res 17:4269

17. O'Brien ME, Socinski MA, Popovich AY, Bondarenko IN, Tomova A, Bilynsky BT, Hotko YS, Ganul VL, Kostinsky IY, Eisenfeld AJ, Sandalic L, Oldham FB, Bandstra B, Sandler AB, Singer JW (2008) Randomized phase III trial comparing singleagent paclitaxel Poliglumex (CT-2103, PPX) with single-agent gemcitabine or vinorelbine for the treatment of PS 2 patients with chemotherapy-naive advanced non-small cell lung cancer. J Thorac Oncol 3:728

18. Omelyanenko V, Gentry C, Kopeckova P, Kopecek J (1998) HPMA copolymer-anticancer drug-OV-TL16 antibody conjugates. II. Processing in epithelial ovarian carcinoma cells in vitro. Int J Cancer 75:600

19. Paz-Ares L, Ross H, O'Brien M, Riviere A, Gatzemeier U, Von Pawel J, Kaukel E, Freitag L, Digel W, Bischoff H, GarciaCampelo R, Iannotti N, Reiterer P, Bover I, Prendiville J, Eisenfeld AJ, Oldham FB, Bandstra B, Singer JW, Bonomi P (2008) Phase III trial comparing paclitaxel poliglumex vs docetaxel in the second-line treatment of non-small-cell lung cancer. Br J Cancer 98:1608

20. Rice JR, Gerberich JL, Nowotnik DP, Howell SB (2006) Preclinical efficacy and pharmacokinetics of AP5346, a novel diaminocyclohexane-platinum tumor-targeting drug delivery system. Clin Cancer Res 12:2248

21. Richards DA, Richards P, Bodkin D, Neubauer MA, Oldham F (2005) Efficacy and safety of paclitaxel poliglumex as first-line chemotherapy in patients at high risk with advanced-stage non-small-cell lung cancer: results of a phase II study. Clin Lung Cancer 7:215 
22. Rowinsky EK, Donehower RC (1995) Paclitaxel (taxol). N Engl J Med 332:1004

23. Sabbatini P, Aghajanian C, Dizon D, Anderson S, Dupont J, Brown JV, Peters WA, Jacobs A, Mehdi A, Rivkin S, Eisenfeld AJ, Spriggs D (2004) Phase II study of CT-2103 in patients with recurrent epithelial ovarian, fallopian tube, or primary peritoneal carcinoma. J Clin Oncol 22:4523

24. Sharma A, Mayhew E, Straubinger RM (1993) Antitumor effect of taxol-containing liposomes in a taxol-resistant murine tumor model. Cancer Res 53:5877

25. Singer JW, Baker B, De Vries P, Kumar A, Shaffer S, Vawter E, Bolton M, Garzone P (2003) Poly-(L)-glutamic acid-paclitaxel (CT-2103) [XYOTAX], a biodegradable polymeric drug conjugate: characterization, preclinical pharmacology, and preliminary clinical data. Adv Exp Med Biol 519:81

26. Sparreboom A, Scripture CD, Trieu V, Williams PJ, De T, Yang A, Beals B, Figg WD, Hawkins M, Desai N (2005) Comparative preclinical and clinical pharmacokinetics of a cremophor-free, nanoparticle albumin-bound paclitaxel (ABI-007) and paclitaxel formulated in Cremophor (Taxol). Clin Cancer Res 11:4136

27. Wang X, Zhao G, Van S, Jiang N, Yu L, Vera D, Howell SB (2009) Pharmacokinetics and tissue distribution of PGG-paclitaxel, a novel macromolecular formulation of paclitaxel, in nu/nu mice bearing NCI-460 lung cancer xenografts. Cancer Chemother Pharmacol [Epub ahead of print] 